\title{
MAPEAMENTO DE REMANESCENTES EM ESTÁGIO INICIAL DE SUCESSÃO NA FLORESTA SUBTROPICAL ATLÂNTICA DO SUL DO BRASIL
}

\section{Mapping Early Successional Stages of the Atlantic Subtropical Rain Forest in Southern Brazil}

\author{
Gustavo Antonio Piazza ${ }^{1}$ \\ Alexander Christian Vibrans ${ }^{12}$ \\ Veraldo Liesenberg ${ }^{3}$ \\ Laio Zimmermann de Oliveira ${ }^{2}$ \\ Paolo Moser 2 \\ 1 Programa de Pós-Graduação em Engenharia Ambiental, Fundação Universidade Regional de \\ Blumenau, Rua São Paulo, 3250, 89.030-000, Blumenau, Santa Catarina, Brasil. E-mail: \\ gustavoapiazza@gmail.com.
}

2 Departamento de Engenharia Florestal, Fundação Universidade Regional de Blumenau, Rua São Paulo, 3250, 89.030-000, Blumenau, Santa Catarina, Brasil. E-mail: acvibrans@gmail.com; laiozoliveira@gmail.com; paolo.moser@gmail.com

${ }^{3}$ Departamento de Engenharia Florestal, Universidade Estadual de Santa Catarina (UDESC), 88520000, Lages, Santa Catarina, Brasil. E-mail: veraldo@gmail.com

\section{Resumo:}

Neste estudo foi abordada a segmentação de imagens de alta resolução e a utilização da técnica da mineração de dados. O objetivo foi encontrar correlação entre resposta espectral, espacial, de contexto e de textura, e variáveis dendrométricas obtidas de inventários florestais em áreas de estágio inicial de sucessão da Floresta Ombrófila Densa em três locais do Estado de Santa Catarina. Os dados de campo foram levantados em seis Unidades Amostrais (UA) com $1.600 \mathrm{~m}^{2}$ cada. No processamento digital utilizaram-se três imagens de alta resolução espacial $(0,39 \mathrm{~m})$ obtidas pelo sensor SAAPI, com três bandas no visível, três no infravermelho próximo, modelo numérico de terreno e de superfície. Dados extraídos do produto digital (atributos) foram utilizados na etapa da mineração dos dados, que selecionou atributos relevantes e descartou aqueles de menor peso. Verificou-se que tanto no estrato arbóreo quanto na regeneração existe heterogeneidade de variáveis, como número de indivíduos $(\mathrm{N})$, diâmetro a altura do peito (DAP) e área basal (AB). Mesmo assim, foram encontradas correlações significativas entre atributos das imagens e os dados de campo. A correlação de maior magnitude absoluta de $\mathrm{N}$ foi com as médias da banda $1(-0,64), 3(-0,62)$ e IR1 $(0,63)$, para DAP foi a razão das bandas IR3 $(0,56)$ e 2 $(0,55)$ e para $A B$ foi o menor valor de pixel das bandas $1(-0,64)$ e IR3 $(-0,60)$, todas altamente significativas $(\mathrm{p}<0,01)$. Esses resultados configuram pontos de partida para futuras investigações a respeito da construção de um estimador de parâmetros biofísicos da vegetação.

Palavras-chave: sucessão; análise da vegetação; sensoriamento remoto. 


\begin{abstract}
:
High-resolution image segmentation and data mining techniques were used in order to find statistical correlation between spatial, spectral, context and texture attributes, considering field data from early stages of secondary forests in the three study sites of Rain Forest in Santa Catarina State, Brazil. Field data were collected in six sampling plots with 1,600 $\mathrm{m}^{2}$ each. Digital processing utilized three high resolution images $(0.39 \mathrm{~m})$ obtained by SAAPI sensor with three visible bands, three near infrared bands, one terrain and one surface model. Extracted data from the digital product (attributes) were used for data mining purposes, selecting the ones with relevance and dismissing those of lesser weight. It was found that both tree and regeneration layers are heterogeneous considering number of individuals $(\mathrm{N})$, diameter at breast height $(\mathrm{DBH})$ and basal area $(\mathrm{AB})$. However, significant correlations between forest attributes and field data were found. The higher correlation of $\mathrm{N}$ is with the average of the band $1(-0.64), 3(-0.62)$ and IR1 (0.63) for DBH was the ratio of bands IR3 (0.56) and $2(0.55)$ and the variable AB was the smallest pixel value of band $1(-0.64)$ and IR3 (-0.60). All correlation coefficients were highly significant $(\mathrm{p}<0.01)$. These results constitute the starting point for future research on the relationship between groups of variables in order to estimate biophysical parameters.
\end{abstract}

Keywords: succession; vegetation analysis; remote sensing.

\title{
1. Introdução
}

Ecossistemas florestais apresentam elevada diversidade biológica e genética (Holdridge, 1947, 1967; Dinerstein et al., 1995). Eles são responsáveis por fornecer habitat para 70\% das espécies de animais e plantas conhecidas (Matthews et al., 2000), contribuir com a produção de biomassa terrestre (Groombridge e Jenkins, 2002) e fornecer benefícios vitais para a economia, sociedade e meio ambiente (Vibrans et al., 2012). Sua estrutura e composição são resultantes da interação de fatores bióticos, abióticos e antrópicos. Há algum tempo, o fator antrópico vem prevalecendo sobre os naturais, fazendo com que a cobertura florestal em várias partes do globo não seja mais contínua, constituindo-se de uma espécie de mosaico vegetal, composto por relictos de florestas em diferentes estágios de degradação. Seguindo o aumento dos fatores antrópicos, ocorre a fragmentação das áreas cobertas por florestas (Tenenbaum et al., 2013).

Dos biomas florestais, as florestas tropicais estão entre a parcela mais ameaçada devido à proximidade de regiões de alta taxa de crescimento demográfico e expansão econômica. Em relação à Floresta Atlântica, restam entre 11 e 16\% da área de domínio deste bioma no Brasil (Ribeiro et al., 2009). No Estado de Santa Catarina, os remanescentes perfazem $28 \%$ da área original (Vibrans et al., 2013). Entretanto, a maior parte de seus remanescentes está representada por fragmentos de florestas secundárias (Siminski et al., 2004; Schuch, 2008, Siminski et al., 2013), sendo poucos núcleos classificados como florestas primárias. Remanescentes florestais conservados em Santa Catarina encontram-se em áreas de altitude, elevada declividade e/ou de difícil acesso (Reis et al. 1995; Liebsch et al. 2008). Prates-Clark et al. (2009) e Silva et al. (2014) detalharam o papel do abandono das áreas de agricultura e pecuária na formação desse mosaico florestal nas regiões tropicais. Essa vegetação "abandonada" tem um papel importante 
no processo de estocagem de carbono e na recuperação de solos (Feldpausch et al. 2004, Orihuela-Belmonte et al., 2013, Lu et al., 2014).

Ao iniciar uma pesquisa sobre mapeamento de florestas secundárias, faz-se necessário conhecer como estas se constituem, ou seja, de que forma ocorre sua regeneração. Uma forma de compreender estes processos de regeneração envolve a análise das mudanças florísticas e estruturais da vegetação nas diversas escalas de tempo (Mantovani, 1998, Teixeira et al., 2009). Estes estudos podem ser realizados por meio de estudos simultâneos das fases de sucessão, como o acompanhamento em campo ou pelo sensoriamento remoto óptico. Levantamentos em campo geralmente são eficientes, pois descrevem informações estruturais da vegetação, porém, cada vez mais, técnicas de processamento digital de dados de sensores remotamente situados vem sendo utilizadas, com objetivo de reduzir custos gerados com as etapas de inventário de campo. Inicialmente, a classificação de imagens por meio destes sensores foi utilizada apenas para mapear áreas desmatadas (Tucker e Townshend, 2000), enquanto que a identificação de florestas secundárias não foi o objetivo principal (Vibrans et al., 2013). Na época existia também uma limitação tecnológica devido à baixa resolução espacial e espectral das imagens, que não eram adequadas para a finalidade proposta. Com o advento de novos sensores e melhorias nas resoluções radiométrica, espectral, espacial e temporal, o cenário mudou, sendo que além das tradicionais classificações de áreas florestadas e não florestadas, hoje os sensores já são usados para classificação de alvos complexos, como florestas secundárias e feições urbanas. Entretanto, mesmo com a melhora das ferramentas de análise digital, observa-se que em Santa Catarina estudos com sensores remotos para a classificação de florestas secundárias mostram-se incipientes, restritos a ferramentas tradicionais e imagens de média resolução espacial (Vibrans et al., 2013). Por exemplo, mapas de estimativa da área coberta por florestas no estado de Santa Catarina, entre 2005 a 2010 (LCF/SAR; PROBIO; Atlas e PPMA) apresentaram resultados discrepantes, variando de $22 \%$ a $41 \%$ (Vibrans et al., 2013). Acredita-se que os responsáveis por estas divergências sejam: (i) a dificuldade de classificar a vegetação nos estágios iniciais devido as deficiências na caracterização espectral; (ii) a dificuldade na padronização de metodologias de classificação, e (iii) a falta de critérios para definição da classe "floresta" (Chazdon et al., 2016). Estas discrepâncias podem ocorrer por razões diversas, dentre estas a incompatibilidade entre resolução espacial do sensor e tamanho das áreas a serem mapeadas e resolução espectral e padrão de altura e densidade da vegetação (Piazza, 2014; Piazza et al., 2016). Considerando as deficiências na caracterização espectral, Zhang e Xie (2012) e Zhang et al. (2013), explicam que sensores com uma resolução especial relativamente "grosseira" (20-30 m ou maior) não conseguem caracterizar todos os tipos de florestas pois a maioria das comunidades são misturas de árvores, arbustos, herbáceas terrestres e áreas antrópicas.

Recentemente, entretanto, múltiplas fontes encontram-se disponíveis para extrair informações detalhadas dos objetos terrestres como modelos digitais de elevação e seus subprodutos (Bradter et al., 2011), dados de levantamentos florísticos, séries temporais (Hüttich et al., 2011) e, recentemente, de LIDAR (Light Detection and Ranging) (Castilho et al., 2012). De acordo com Wulder et al. (2004), Johansen e Pihnn (2006), Wang et al. (2007) e Johansen et al. (2007), é possível estimar parâmetros estruturais da vegetação, com uso de sensores adequados.

Seguindo esta mesma ideia de pesquisa, o objetivo deste trabalho é verificar se existe relação entre variáveis dendrométricas e resposta espectral de áreas cobertas por estágios iniciais da sucessão na Floresta Ombrófila Densa em Santa Catarina. Para tanto, foram mensuradas variáveis dendrométricas em seis unidades amostrais (UA) da vegetação e avaliadas as respostas espectrais em imagens de alta resolução espacial. 


\subsection{Estágios sucessionais da Floresta Ombrófila Densa}

Sucessão é o mecanismo pelo qual florestas se renovam naturalmente quando perturbadas (Gómez-Pompa, 1971). De forma geral, florestas são classificadas como "maduras", quando apresentam alta de diversidade e complexidade, altos valores de área basal, volume de madeira e biomassa, dossel fechado e baixo grau de atividades antrópicas recentes. São chamadas "secundárias", florestas que sofreram perturbação natural e/ou humana recente, seja em um único momento ou durante um período prolongado, e apresentam diferenças significativas de composição e estrutura de espécies quando comparadas com florestas próximas (Chokkalingam e Jong, 2001).

A sequência e a duração dos estágios sucessionais varia substancialmente entre as tipologias florestais (Chazdon, 2008). Utilizam-se diferentes técnicas de classificação destes estágios. Siminski (2009), por exemplo, cita cinco diferentes abordagens de classificação de estágios sucessionais da vegetação secundária, envolvendo: idade da vegetação, características fisionômicas, parâmetros estruturais, análises multivariadas e sensoriamento remoto. Geralmente são classificadas em: (i) estágio inicial, com presença de plantas herbáceas, samambaias, gramíneas, arbustos, lianas, trepadeiras e cipós (Budowski, 1965; Ewell, 1983; Finegan, 1996). (ii) Estágio médio, conhecido como estágio de arvoretas (Siminski, 2004), caracterizado pelo aparecimento de espécies lenhosas de pequeno porte com copas estreitas, que formam microclima úmido sombreado. Segundo Oliver e Larson (1990), esta etapa é conhecida como "fase de exclusão", devido ao aumento da altura das árvores colonizadoras que ocasionam o fechamento do dossel e diminuem a disponibilidade de luz no sub-bosque, proporcionando o desaparecimento de espécies exigentes de luminosidade e o surgimento de árvores tolerantes à sombra, como algumas palmeiras e xaxins (Chazdon, 2008). (iii) Estágio avançado, também denominado mata secundária (Klein, 1980), mostrando certas semelhanças fisionômicas com as florestas maduras, embora ainda sem a dominância de espécies tolerantes, chamadas "climácicas", regenerantes na sombra, de crescimento lento, ciclo de vida longo, madeira densa e de alto valor comercial.

\section{Materiais e métodos}

\section{1 Área de estudo}

Foram escolhidas três áreas de estudo (A, B e C), inseridas na fitofisionomia da Floresta Ombrófila Densa (FOD), no litoral centro norte do Estado, em áreas de ocorrência de estágios iniciais de sucessão em meio a agriculta e pecuária. 


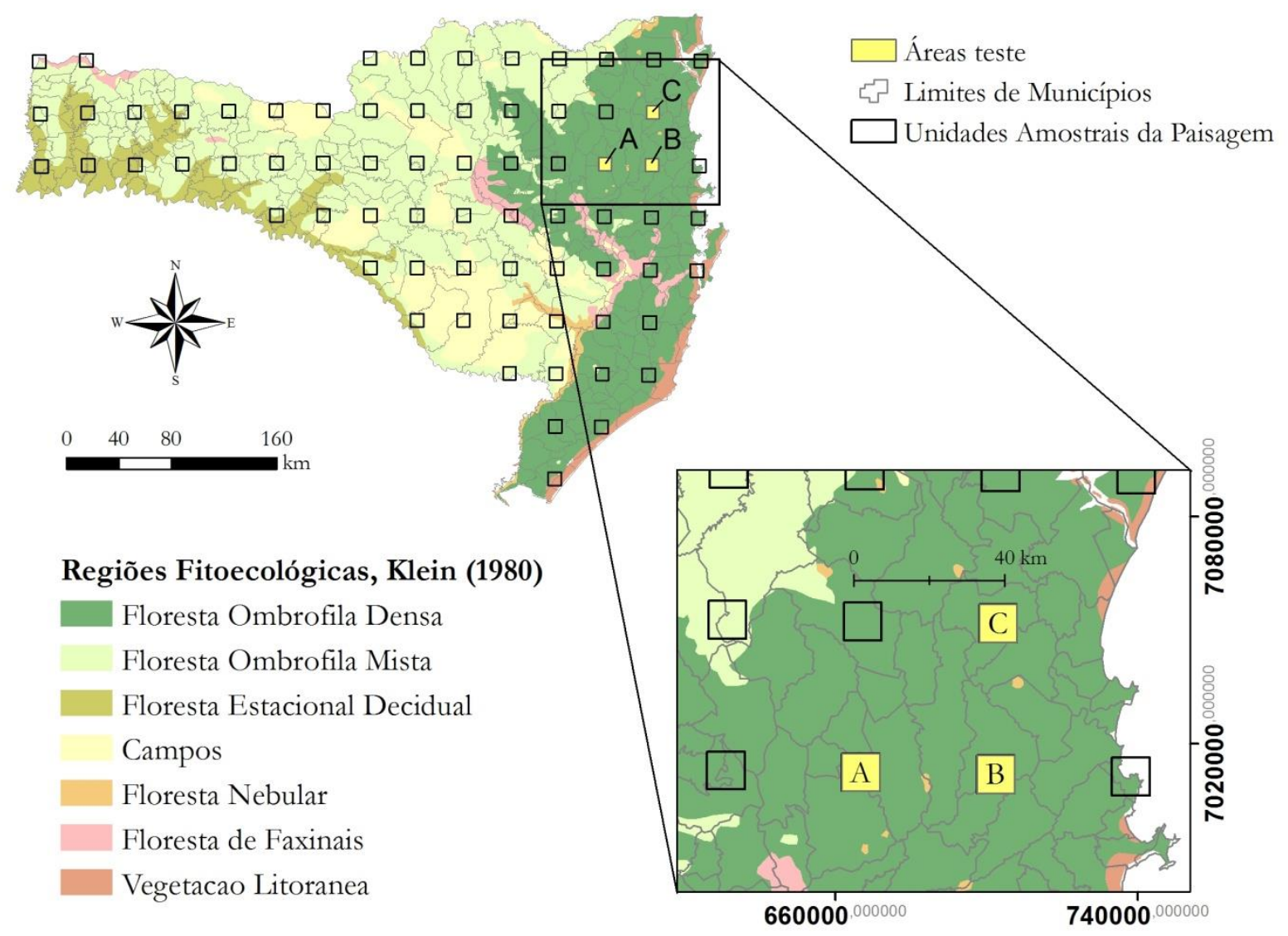

Figura 1: Localização das três áreas teste para com o estado de Santa Catarina, Sul do Brasil.

\subsection{Levantamento em campo}

Dentro das áreas testes (A, B e C), foram instaladas três Unidades Amostrais (UA). Diferentes quantidades de UA foram instaladas nas áreas teste. Três (03) UA na área A, uma (01) UA na área B e duas (02) UA na área C. A alocação das UA ocorreu de acordo com a ocorrência das áreas de vegetação em estágio inicial. A estrutura da UA baseou-se na utilizada pelo IFFSC (Inventário Florístico Florestal de Santa Catarina), que utiliza a metodologia dos conglomerados compostos por quatro subunidades perpendiculares a um ponto central (Vibrans et al., 2010). A área total do conglomerado utilizado neste estudo é de $1.600 \mathrm{~m}^{2}$, com quatro subunidades de 400 $\mathrm{m}^{2}$. As subunidades têm dimensões de $20 \mathrm{~m}$ x $20 \mathrm{~m}$ e são orientadas na direção dos pontos cardeais (norte, sul, leste e oeste). Cada subunidade encontra-se a $10 \mathrm{~m}$ do centro e é dividida em quatro quadrantes de $10 \mathrm{~m}$ x $10 \mathrm{~m}\left(100 \mathrm{~m}^{2}\right)$ (Figura 2). Em cada quadrante foi anotado o nome científico, a circunferência à altura do peito (CAP - 1,3 $\mathrm{m}$ do solo) e a altura média de todos os indivíduos com CAP $\geq 9,42 \mathrm{~cm}$ (ou diâmetro a altura do peito, DAP, $\geq 3 \mathrm{~cm}$ ). Visando contemplar o estrato que não atendeu ao critério de inclusão, foram alocadas subparcelas de $5 \mathrm{~m}$ x $5 \mathrm{~m}\left(25 \mathrm{~m}^{2}\right)$ nas extremidades do polígono imaginário, sendo um total de 4 subparcelas por subunidade (Figura 2). Nas 16 subparcelas os indivíduos foram contatos e as espécies foram registradas; a altura média geral dos indivíduos também foi coletada. 


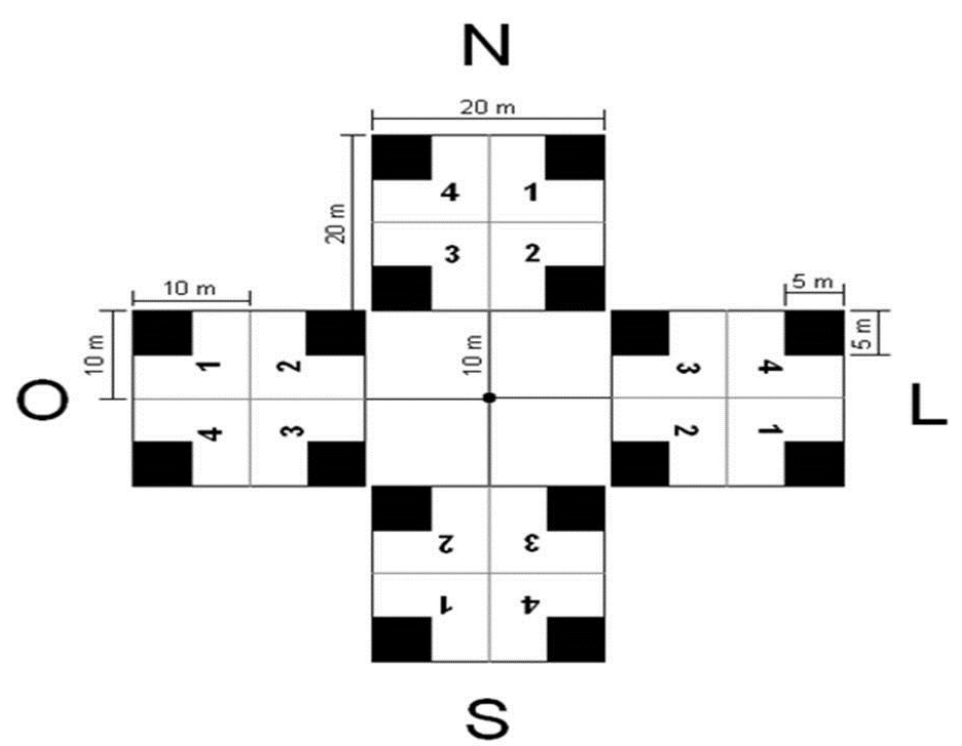

Figura 2: Estrutura da UA de levantamento em campo

\subsection{Levantamento em campo}

A análise de variância (ANOVA) e o teste post hoc de Tukey-Kramer foram aplicados para testar a igualdade das médias das variáveis dendrométricas (número de indivíduos - $\mathrm{N}$, diâmetro a altura do peito - DAP e área basal - AB) entre as UA de cada área, baseada na variabilidade das subunuidades. Todas as análises utilizaram significância de $\alpha=0,05$ utilizando o software PAST versão 2.14 .

Para verificar a similaridade entre as UA foram utilizados dados de composição de espécies e a técnica multivariada da análise de agrupamentos. A análise de agrupamentos utilizou o algoritmo de ligação de Ward. As medidas de similaridade foram Bray-Curtis que utiliza valores de abundância de espécies e Jaccard que utiliza dados de presença/ausência ( 0 ou 1). Foram calculados também os índices de similaridade (Sørensen) e diversidade (Simpson [1-D] e Shannon [H']).

\subsection{Correlação dos dados de campo com imagens}

Os dados digitais de entrada foram as imagens do aerolevantamento fotográfico do Estado de Santa Catarina, executado pela Secretaria de Estado do Desenvolvimento Econômico Sustentável (SDS-SC) em 2011 por meio de um Sistema Aerotransportado de Aquisição e Pósprocessamento de Imagens Digitais (SAAPI). As imagens são compostas por ortofotos multiespectrais (banda azul: 0,38 - 0,52 $\mu \mathrm{m}$; verde: $0,52-0,63 \mu \mathrm{m}$; vermelho: $0,63-0,70 \mu \mathrm{m}$ ) e imagens no infravermelho próximo (NIR) (IR1: 0,76 $\mu \mathrm{m}$; IR2: 0,77 $\mu \mathrm{m}$; IR3: 0,78 $\mu \mathrm{m}$ ), com resolução espacial de 0,39 m e resolução radiométrica de 8 bits (ENGEMAP, 2012). Além das ortofotos foram disponibilizados também um modelo numérico de terreno e superfície, no qual foi usado para criar o atributo altura. 
As imagens foram processadas nos softwares eCognition 8, ArcGIS 10.3 e WEKA 3.6. Inicialmente no eCognition imagens foram segmentadas por meio do algoritmo multiresolução que criou objetos de acordo com a resposta da imagem. A segmentação utilizou a combinação de parâmetros: 0,2 de forma, 0,3 de compacidade e 180 de escala. Manualmente foi feita uma seleção de amostras de treinamento para as 10 classes de uso do solo, posteriormente utilizadas na exportação da tabela de atributos com aproximadamente 80 atributos para todas as UA. A tabela foi importada para o software de mineração de dados (WEKA 3.6) onde foi feita a etapa de seleção dos atributos por meio do método do ranqueamento que ordena os dados de acordo com sua relevância. No sentido de verificar diferenças entre o refinamento do processo de seleção de atributos, foram selecionados 12 atributos para a área teste $\mathrm{A}, 23$ para a área teste $\mathrm{B}$ e 73 para a área teste $\mathrm{C}$. O conjunto de dados de campo (N, AB, DAP) e de atributos foi submetido a análise de normalidade por meio do QQ-plots. Como os conjuntos de dados analisados não apresentaram distribuição normal utilizou-se o coeficiente de correlação não paramétrico de Spearman. A significância das correlações foi testada para $\alpha=0,01$.

\section{Resultados}

\subsection{Inventários de campo}

Na Tabela 1 constam, em ordem decrescente, as dez espécies mais abundantes em cada uma das UA.

Tabela 1: Espécies mais abundantes por UA no estrato arbóreo das áreas teste, Santa Catarina, Brasil

\begin{tabular}{|c|c|c|c|c|c|c|}
\hline & UAl & UA2 & UA3 & UA4 & UA5 & UA6 \\
\hline 1 & IM & $\begin{array}{l}\text { Myrsine } \\
\text { coriacea }\end{array}$ & $\begin{array}{l}\text { Cyathea } \\
\text { atrovirens }\end{array}$ & Myrsine coriacea & $\begin{array}{l}\text { Aegiphila } \\
\text { integrifolia }\end{array}$ & $\begin{array}{c}\text { Miconia } \\
\text { cinnamomifolia }\end{array}$ \\
\hline 2 & $\begin{array}{c}\text { Miconia } \\
\text { cinnamomifolia }\end{array}$ & $\begin{array}{c}\text { Andira } \\
\text { fraxinifolia }\end{array}$ & $\begin{array}{l}\text { Myrsine } \\
\text { coriacea }\end{array}$ & Miconia cabucu & $\begin{array}{l}\text { Vernonanthura } \\
\text { divaricata }\end{array}$ & $\begin{array}{l}\text { Tibouchina } \\
\text { urvilleana }\end{array}$ \\
\hline 3 & $\begin{array}{l}\text { Piptocarpha } \\
\text { axillaris }\end{array}$ & Psidium guajava & $\begin{array}{c}\text { Miconia } \\
\text { cinnamomifolia }\end{array}$ & $\begin{array}{l}\text { Cecropia } \\
\text { glaziovii }\end{array}$ & Casearia sylvestris & $\begin{array}{l}\text { Casearia } \\
\text { sylvestris }\end{array}$ \\
\hline 4 & $\begin{array}{l}\text { Myrsine } \\
\text { coriacea }\end{array}$ & Pinus taeda & IM & IM & NI 1 & Baccharis sp.1 \\
\hline 5 & $\begin{array}{l}\text { Casearia } \\
\text { sylvestris }\end{array}$ & $\begin{array}{l}\text { Tibouchina } \\
\text { urvilleana }\end{array}$ & Psidium guajava & $\begin{array}{l}\text { Baccharis } \\
\text { semiserrata }\end{array}$ & $\begin{array}{l}\text { Aspidosperma } \\
\text { australe }\end{array}$ & Psidium guajava \\
\hline 6 & $\begin{array}{l}\text { Schefflera } \\
\text { morototoni }\end{array}$ & $\begin{array}{c}\text { Schinus } \\
\text { terebinthifolius }\end{array}$ & $\begin{array}{c}\text { Schinus } \\
\text { terebinthifolius }\end{array}$ & $\begin{array}{l}\text { Tibouchina } \\
\text { urvilleana }\end{array}$ & $\begin{array}{l}\text { Vernonanthura } \\
\text { puberula. }\end{array}$ & IM \\
\hline 7 & Pinus taeda & - & $\begin{array}{c}\text { Xylopia } \\
\text { brasiliensis }\end{array}$ & $\begin{array}{c}\text { Hieronyma } \\
\text { alchorneoides }\end{array}$ & Myrsine coriacea & $\begin{array}{l}\text { Cupania } \\
\text { vernalis }\end{array}$ \\
\hline 8 & Baccharis sp.1 & - & $\begin{array}{l}\text { Tibouchina } \\
\text { urvilleana }\end{array}$ & $\begin{array}{c}\text { Austroeupatorium } \\
\text { sp. } 1\end{array}$ & $\begin{array}{c}\text { Tabernaemontana } \\
\text { catharinensis }\end{array}$ & Miconia cabucu \\
\hline 9 & $\begin{array}{c}\text { Myrcia } \\
\text { hebepetala }\end{array}$ & - & $\begin{array}{c}\text { Pera } \\
\text { glabrata }\end{array}$ & $\begin{array}{c}\text { Nectandra } \\
\text { membranacea }\end{array}$ & Annona sp. & $\begin{array}{c}\text { Miconia } \\
\text { sp.1 }\end{array}$ \\
\hline 10 & $\begin{array}{l}\text { Cyathea } \\
\text { atrovirens }\end{array}$ & - & Miconia cabucu & Cyathea atrovirens & - & Baccharis sp.2 \\
\hline
\end{tabular}

* Legenda: NI - não identificada; IM - indivíduos mortos. 
As espécies características do estágio inicial de sucessão ocorrem com os maiores valores de cobertura (VC) em todas as UA, como por exemplo, Miconia cinammomifolia, Myrsine coriacea, Baccharis sp., Tibouchina urvilleana, Miconia cabucu, Casearia sylvestris, também encontradas por Siminski (2004) e Siminski et al. (2013). Em quatro UA, as árvores mortas estavam entre as espécies mais abundantes mostrando que as áreas em regeneração estão em processo dinâmico de ocupação.

Na Tabela 2 são apresentados os resultados dos inventários das UA para o estrato arbóreo.

Tabela 2: Estimativas das variáveis dendrométricas do estrato arbóreo das UA das áreas teste, com intervalo de confiança $(\alpha=0,05)$, Santa Catarina, Brasil

\begin{tabular}{c|c|c|c|c|c|c}
\hline $\mathbf{U A}^{\mathbf{1}}$ & $\mathbf{A T}$ & $\mathbf{N}^{\mathbf{2}}$ & N.ha $^{-\mathbf{1 ~ 2}}$ & $\mathbf{A B}^{\mathbf{2}}$ & AB.ha $^{-\mathbf{~ 2}}$ & DAP $^{\mathbf{3}}$ \\
\hline 01 & A & $103 \pm 3,8$ & $643,7 \pm 23,8$ & $0,68 \pm 0,03$ & $4,26 \pm 0,2$ & $7,06 \pm 2,1$ \\
\hline 02 & A & $82 \pm 21,8$ & $512,5 \pm 135,9$ & $0,16 \pm 0,03$ & $1,02 \pm 0,2$ & $6,21 \pm 6,0$ \\
\hline 03 & A & $134 \pm 6,6$ & $837,5 \pm 41,6$ & $0,75 \pm 0,07$ & $4,72 \pm 0,4$ & $5,26 \pm 1,2$ \\
\hline 04 & C & $260 \pm 10,8$ & $1625,0 \pm 67,5$ & $0,66 \pm 0,04$ & $7,24 \pm 0,3$ & $8,16 \pm 2,6$ \\
\hline 05 & C & $30 \pm 3,2$ & $187,5 \pm 20,1$ & $0,23 \pm 0,03$ & $1,46 \pm 0,2$ & $8,87 \pm 3,6$ \\
\hline 06 & B & $205 \pm 3,9$ & $1281,2 \pm 24,6$ & $0,54 \pm 0,01$ & $3,38 \pm 0,05$ & $5,62 \pm 0,8$ \\
\hline
\end{tabular}

* Legenda: ${ }^{1}$ unidade amostral com quatro subunidades $;{ }^{2}$ soma e ${ }^{3}$ média; AT = área teste; $\mathrm{N}=$ número de indivíduos por unidade amostral; ha = hectare; $\mathrm{AB}=$ área basal $\left(\mathrm{m}^{2}\right) ; \mathrm{DAP}=$ diâmetro a altura do peito $(\mathrm{cm})$.

De acordo com os resultados da ANOVA, todas as variáveis analisadas apresentaram diferenças significativas entre as subunidades. Ou seja, todas as UA são representadas por vegetação não homogênea. Estes resultados podem ser, ao menos em parte, confirmados mediante a interpretação visual dos respectivos recortes das imagens (Figura 3).
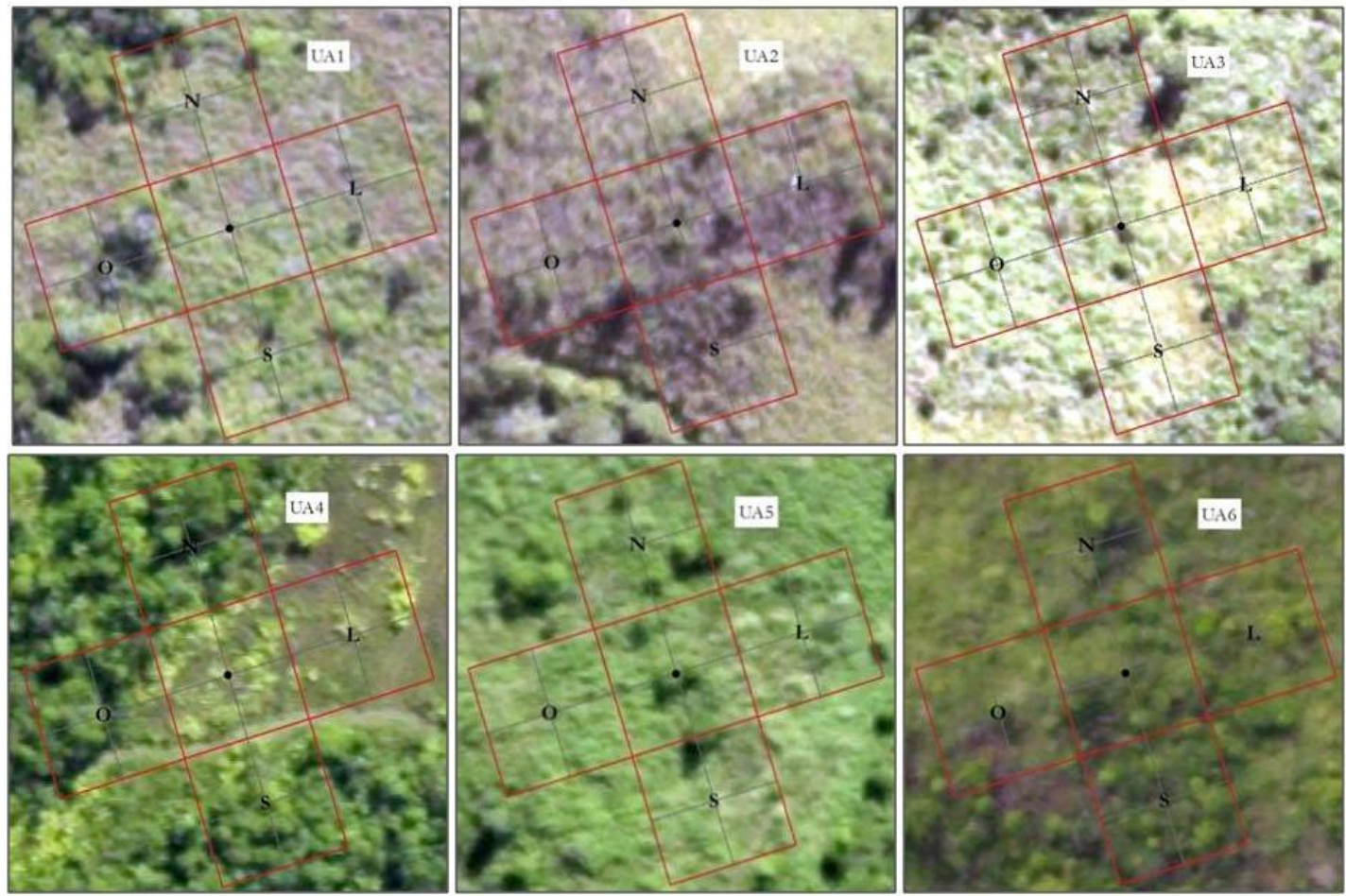

Figura 3: Recorte das imagens de alta resolução espacial adquirida pelo sensor SAAPI (2011) na composição 321 (RGB) e com suas respectivas UA, áreas teste em Santa Catarina, Brasil 


\subsection{Similaridade entre UAs}

A análise de agrupamento formou dois grupos distintos (Figura 4), utilizando o valor 500 como linha de corte (Souza et al., 1990). Um grupo com as UA 4 e 6, que foram aquelas que apresentaram os maiores valores de $\mathrm{N}$ e $\mathrm{AB}$; outro grupo composto pelas UA 5 e 1, 2 e 3), estas últimas localizadas na mesma área teste.

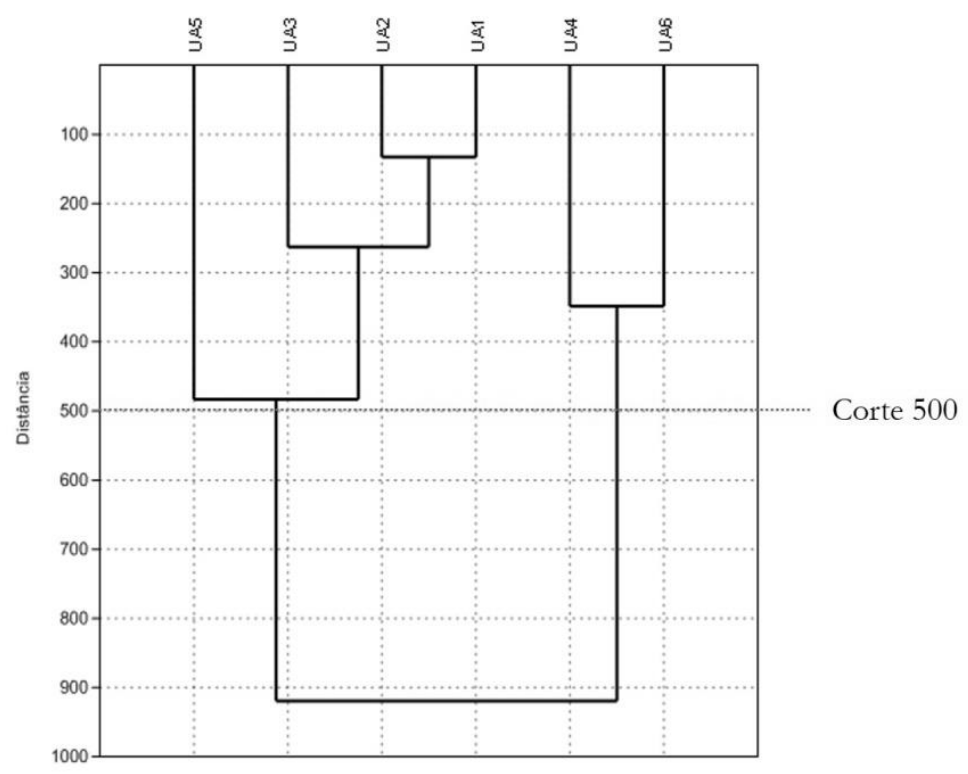

Figura 4: Análise de agrupamento com variáveis dendrométricas entre as UA $(\alpha=0,05)$, áreas teste em Santa Catarina, Brasil

O agrupamento pela medida de similaridade de Bray-Curtis gerou um coeficiente cofenético de 0,9288 e pelo índice de Jaccard de 0,8662, demonstrando a confiabilidade da análise. Por BrayCurtis a maior similaridade de espécies no estrato arbóreo foi em relação a UA 2 e 3 (próximas em distância). As UA 2 e 3 também apresentaram similaridade com a UA 4 (maior valor de N). Em relação ao índice de Jaccard, a UA 4 e 6 foram as que apresentaram maior similaridade de espécies do estrato arbóreo, em seguida agruparam-se a UA 3, 1 e 2. Quantificou-se também a riqueza e os valores dos índices de diversidade de Simpson D- e Shannon H-, calculados por UA, mostram que a maior diversidade de espécie foi encontrada na UA $6(0,8674$ e 2,443) e a menor na UA $2(0,5137$ e 0,9805) condizentes com a riqueza de espécies observada.

\subsection{Regeneração}

Os inventários de campo para a regeneração estão resumidos na Tabela 3. 
Tabela 3: Estimativas das variáveis dendrométricas por UA para indivíduos da regeneração com intervalo de confiança $(\alpha=0,05)$, Santa Catarina, Brasil

\begin{tabular}{c|c|c}
\hline UA & $\mathbf{N}^{\mathbf{1}}$ & N.ha $^{\mathbf{1}} \mathbf{1}$ \\
\hline 01 & $586 \pm 7,6$ & $14650 \pm 189,98$ \\
\hline 02 & $850 \pm 28,7$ & $21250 \pm 717,19$ \\
\hline 03 & $265 \pm 6,7$ & $6625 \pm 168,05$ \\
\hline 04 & $350 \pm 4,9$ & $8750 \pm 123,02$ \\
\hline 05 & $30 \pm 1,0$ & $750 \pm 24,74$ \\
\hline 06 & $136 \pm 0,9$ & $3400 \pm 23,17$ \\
\hline
\end{tabular}

* Legenda: ${ }^{1}$ soma; $\mathrm{N}=$ número de indivíduos na unidade amostral; e ha = hectare.

A ANOVA mostrou a homogeneidade das UA em relação à média da variável $\mathrm{N}$; sendo que todas as subunidades aceitaram a hipótese de igualdade para $\mathrm{N}$.

\subsection{Análise dos dados digitais}

Na figura 5 são apresentadas as médias da resposta espectral das dez classes de uso do solo analisadas, com os seus respectivos desvios padrões. Nota-se que as classes da vegetação possuem comportamento espectral similar. Os padrões encontrados estão de acordo com os reportados em Meneses e Netto (2002). As classes solo exposto e edificações (número digital [ND] elevado na banda 1, baixo na banda 3 e estável no infravermelho) apresentaram alta variabilidade e não foram difíceis de caracterizar. Já as classes relacionadas à vegetação tiveram comportamento espectral muito próximo quanto aos ND. Ocorre para estas classes a sobreposição dos desvios padrões dos ND indicando similaridade espectral. As classes iniciais de regeneração são as que apresentaram maiores valores de ND ao longo do espectro em função da estruturação mais regular dos dosséis. Já para as classes avançadas, observa-se o contrário, uma maior irregularidade dos dosséis e, portanto, maior influência de sombras (Ponzoni et al., 2012). Os padrões espectrais dos diferentes estágios sucessionais estão de acordo com aqueles encontrados por Vieira et al. (2003) e Liesenberg et al. (2010).

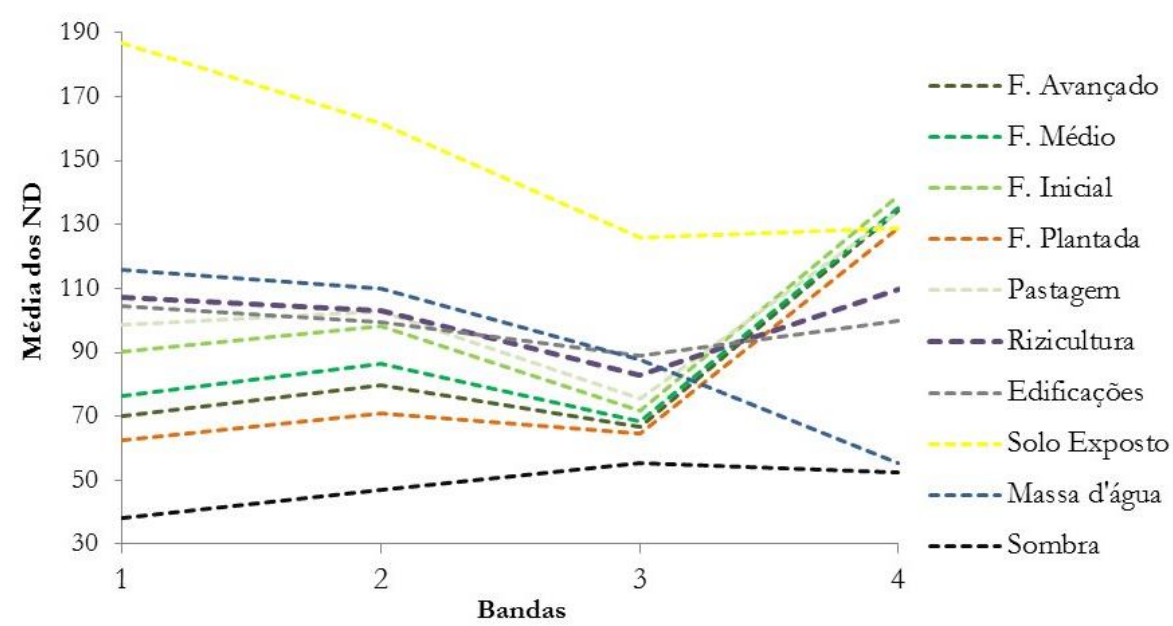

Figura 5: Perfil espectral de amostras de classes de uso do solo para as bandas 1, 2, 3 e 4, na área teste A, Santa Catarina, Brasil 
Com este resultado, evidencia-se a similaridade dos estágios sucessionais estudados em termos de resposta espectral e a necessidade do uso de atributos para a discriminação das mesmas, como textura e forma.

\subsection{Correlação entre atributos e parâmetros estruturais da vegetação}

Foi avaliada a relação entre as variáveis N, DAP e AB, mensuradas em campo e 87 atributos (estatísticos, espaciais e texturais) da vegetação, obtidos das imagens analisadas. Apenas atributos com coeficientes de correlação superior a $\pm 0,4$ foram apresentados na Tabela 4.

Tabela 4: Valores de correlação dos atributos estatísticos, espaciais e texturais em relação aos dados de campo $(\alpha=0,01)$ referentes ao sensor SAAPI (2011), Santa Catarina, Brasil

\begin{tabular}{|c|c|c|c|c|c|c|c|c|}
\hline \multirow[b]{2}{*}{ Atributo } & \multicolumn{2}{|l|}{$\mathbf{N}$} & \multicolumn{3}{|c|}{ DAP } & \multicolumn{3}{|c|}{$\mathbf{A B}$} \\
\hline & Banda & Valor & Atributo & Banda & Valor & Atributo & Banda & Valor \\
\hline Média & 1 & $-0,64$ & NDVI & - & 0,41 & NDVI & - & 0,44 \\
\hline Média & 2 & $-0,48$ & Média & IR1 & 0,52 & Média & 1 & $-0,51$ \\
\hline Média & 3 & $-0,62$ & Razão & 2 & 0,55 & Média & 3 & $-0,50$ \\
\hline Média & IR2 & $-0,63$ & Razão & 3 & 0,42 & Média & IR2 & $-0,50$ \\
\hline Média & IR3 & $-0,46$ & Razão & IR1 & 0,54 & Brilho & - & $-0,44$ \\
\hline $\begin{array}{l}\text { Menor valor } \\
\text { de pixel }\end{array}$ & 1 & $-0,44$ & Razão & IR3 & 0,56 & Razão & IR1 & 0,50 \\
\hline $\begin{array}{l}\text { Menor valor } \\
\text { de pixel }\end{array}$ & 2 & $-0,43$ & $\begin{array}{l}\text { Menor valor } \\
\text { de pixel }\end{array}$ & $\mathrm{R}$ & $-0,49$ & $\begin{array}{l}\text { Menor valor } \\
\text { de pixel }\end{array}$ & 1 & $-0,64$ \\
\hline $\begin{array}{l}\text { Menor valor } \\
\text { de pixel }\end{array}$ & IR2 & $-0,42$ & $\begin{array}{l}\text { Menor valor } \\
\text { de pixel }\end{array}$ & $\mathrm{IR} 2$ & $-0,42$ & $\begin{array}{l}\text { Menor valor } \\
\text { de pixel }\end{array}$ & 3 & $-0,50$ \\
\hline $\begin{array}{l}\text { Menor valor } \\
\text { de pixel }\end{array}$ & IR3 & $-0,41$ & $\begin{array}{l}\text { Maior valor } \\
\text { de pixel }\end{array}$ & IR1 & 0,42 & $\begin{array}{l}\text { Menor valor } \\
\text { de pixel }\end{array}$ & $\mathrm{IR} 2$ & $-0,60$ \\
\hline GLCM StdDev & 2 & 0,45 & $\begin{array}{l}\text { Número de } \\
\text { objetos escuros }\end{array}$ & IR1 & 0,43 & $\begin{array}{l}\text { Menor valor } \\
\text { de pixel }\end{array}$ & IR3 & $-0,47$ \\
\hline GLCM StdDev & IR1 & 0,49 & & & & $\begin{array}{l}\text { Número de } \\
\text { objetos escuros }\end{array}$ & 1 & 0,50 \\
\hline GLCM StdDev & IR3 & 0,47 & & & & $\begin{array}{l}\text { Número de } \\
\text { objetos escuros }\end{array}$ & 3 & 0,45 \\
\hline GLCM Contrast & IR2 & $-0,41$ & & & & $\begin{array}{l}\text { Número de } \\
\text { objetos escuros }\end{array}$ & $\mathrm{IR} 2$ & 0,50 \\
\hline GLCM Correlação & IR2 & 0,43 & & & & & & \\
\hline GLCM Correlação & IR3 & 0,46 & & & & & & \\
\hline
\end{tabular}

A correlação de maior magnitude absoluta da variável $\mathrm{N}$ foi com as médias da banda $1(-0,64), 3$ $(-0,62)$ e IR1 $(0,63)$, para a variável DAP foi a razão das bandas IR3 $(0,56)$ e $2(0,55)$ e para a variável $A B$ foi o menor valor de pixel das bandas $1(-0,64)$ e IR3 $(-0,60)$, todas altamente significativas $(\mathrm{p}<0,01)$. 


\section{Discussão}

Observou-se similaridade em relação à composição de espécies entre as UA no estrato arbóreo, no entanto, dados quantitativos (N, DAP e AB) dessas áreas variaram estatisticamente mesmo entre áreas próximas. Essa heterogeneidade apenas confirma a dificuldade de classificação dessas áreas por meio de imagens aéreas. Cenário que piora quando se trata de média resolução espacial que, em geral, não apresenta resolução espectral suficiente para diferenciar estágios sucessionais de vegetação e florestas plantadas. Dados da regeneração, por outro lado, mostraram maior homogeneidade em relação às espécies e ao número de indivíduos, diferentemente do estrato arbóreo.

Essa heterogeneidade do campo aponta para o principal fator discrepante dos resultados dos levantamentos da cobertura florestal do Estado (LCF/SAR, PROBIO, Atlas 2008 e PPMA/FATMA), que tiveram como método a classificação visual que sofre com interferências de intérpretes. Vale ressaltar que considerando as variáveis dendrométricas, as UA mostram diferenças significativas entre si, embora sejam espectralmente similares. Nestes casos, outros atributos das imagens poderiam ser utilizados para realçar essa diferenciação. Os atributos que mais se assemelharam com os dados de campo foram atributos estatísticos (média, brilho e razão). Analisando a textura de uma imagem de alta resolução espacial para classificação dos estágios sucessionais de Floresta Atlântica no Sul da Bahia, Sette (2009) encontrou significância na relação entre os atributos, Entropia e Momento Diferencial Inverso (MDI) e DAP médio das árvores. Os atributos encontrados neste trabalho (média, brilho e razão) também foram significativos no estudo de Thenkabail et al. (2000), Gong et al. (2003) e Stagakis et al. (2010), que encontraram correlação com atributos biofísicos de culturas agrícolas. Os resultados também são similares a aqueles obtidos por Steininger (2000) que encontrou valores de $\mathrm{r}$ de -0.23 (na banda do vermelho, LANDSAT-TM) e - 0.46 (banda do infravermelho próximo) quando estudou biomassa sobre o solo em uma área próxima de Manaus.

Esses resultados configuram pontos de partida para futuras investigações a respeito da relação entre os grupos de variáveis. Esses atributos poderiam ser explorados no sentido de desenvolver um estimador de parâmetros estruturais da vegetação e predizer características biofísicas da vegetação a partir dos dados de imagens de alta resolução espacial. Como recomendação para trabalhos futuros, sugere-se maiores cuidados em relação a radiometria do sensor em projetos de larga escala. Além da recomendação de fazer uma correção atmosférica dos ND assim como sua conversão radiométrica em reflectância de superfície para realçar os valores de significância obtidos e possibilitar a comparação em uma mesma grandeza física.

\section{Conclusão}

Com base nos resultados apresentados, pode se chegar às seguintes conclusões: i) imagens de alta resolução espacial possibilitam reconhecer padrões espectrais, espaciais e texturais das feições terrestres. ii) a mineração de dados (seleção de atributos) foi decisiva para a seleção dos atributos de maior importância, pois trabalhou com uma extensa base de dados de forma rápida, ranqueando os mais relevantes, descartando aqueles com menor peso. Outra vantagem foi a automatização dos processamentos diminuindo a interferência da subjetividade do intérprete. iii) com este resultado, evidencia-se a similaridade da resposta espectral dos estágios sucessionais 
estudados e a necessidade do uso de outros atributos para a discriminação das mesmas, como textura e forma. iv) foram encontradas relações altamente significativas entre variáveis dendrométricas de campo e atributos espectrais, espaciais e de textura. v) este resultado em parte, é um ponto de partida para desenvolver um estimador de parâmetros biofísicos da vegetação por meio de atributos digitais obtidos em imagens de aerolevantamento e de satélite.

\section{AGRADECIMENTOS}

Os autores agradecem a CAPES pela concessão da bolsa de mestrado do o primeiro autor, à FAPESP (Processo 13/05081-9) pela concessão da bolsa do terceiro autor e ao CNPq pela bolsa de produtividade de pesquisa para o segundo autor.

\section{REFERÊNCIAS BIBLIOGRÁFICAS}

Bradter, Ute, Thom, Tim. J., Altringham, John D., Kunin, William E., and Benton, Tim G. "Prediction of National Vegetation Classification communities in the British uplands using environmental data at multiple spatial scales, aerial images and the classifier random forest." Journal of Applied Ecology 48(4) (2011): 1057-1065.

Budowski, Gerardo. "Distribution of tropical American rain forest species in the light of sucessional processes" Turrialba 15 (1965), 40-42.

Castilho, Mauricio, Benoit, Rrivard, Sanchez-Azofeifa Arturo, Calvo-Alvarado, Julio, and Dubayah, Ralph. "LIDAR remote sensing for secondary Tropical Dry Forest identification". Remote sensing of environment 121 (2012), 131-143.

Chazdon, Robin L. "Change and determinism in Tropical Forest Succession." In Tropical Forest Community Ecology edited by Carson, Walter P., and Schnitzer, Stefean A., 384-408. Oxford: John Wiley \& Sons Ltd, 2008.

Chazdon, Robin L., Brancalion, Pedro H. S., Laestadius, Lars, Bennett-Curry, Aoife, Buckingham, Kathleen, Kumar, Chetan, Moll-Rocek, Julian, Vieira, Ima C. G., and Wilson, Sarah J. "When is a forest a forest? Forest concepts and definitions in the era of forest and landscape restoration." Ambio (2016), 1-13.

Chokkalingam, Unna, Jong Will. D. "Secondary forest: a working definition and typology." International forestry Review, 3(1) (2001), 19-26.

Dinerstein, Eric, Olson, David M., Graham, Douglas J., Webster, Avis L., Primm, Steven A., Bookbinder, Marnie P., Ledec, George, and World Wildlife Fund. "Una evaluacíon del estado de conservacíon de las ecoregiones terrestres de América Latina y el Caribe." Washington D.C.: Fondo Mundial para la Naturaleza, 1995.

ENGEMAP Geoinformação. Relatório de produção final - edital de concorrência pública ${ }^{\circ}$ 0010/2009. Florianópolis SC. 218p, 2012

Ewel, John. J. "Succession.” In: Tropical rain forest ecosystems edited by Golley, F. B., 217233. Amsterdam: Elselvier Scientific Publishing Co, 1983.

Feldpausch, Ted R., Rondon, Marco A., Fernandes, Erick C. M., Riha, Susan J., and Wandelli, Elisa. "Carbon and Nutrient Accumulation in Secondary Forests Regenerating on Pastures in Central Amazonia.” Ecological Applications 14 (2004), 164-176. 
Finegan, Bryan. "Pattern and process in neotropical secondary forests: the first 100 years of sucession." Trends in Ecology and Evolution 11 (1996), 119-124.

Gómez-Pompa, Arturo. "Possible papel de la vegetación secundária en la evolución de la flora tropical." Biotropica 3 (1971), 125-135.

Gong, Peng, Pu, Ruiliang, Biging, Greg S., and Larrieu, Mirta R. "Estimation of Forest Leaf Area Index Using Vegetation Indices Derived From Hyperion Hyperspectral Data." IEEE Transactions on Geoscience and Remote Sensing 41 (2003), 1355-1362.

Groombridge, Brian, and Jenkins, Martin D. World atlas of biodiversity. Berkeley, California: University of California Press, 340p, 2002.

Holdridge, Leslie R. Determination of world plant formations from simple climatic data. Science 105 (1947), 367-368.

Holdridge, Leslie R. Life zone Ecology. San Jose, CA: Tropical Science Center, 60-77, 1967.

Hüttich, Christian, Herold, Martin, Wegmann, Martin, Cord, Anna, Strohbach, Ben, Schmullius, Christiane, and Dech, Stefan "Assessing effects of temporal compositing and varying observation periods for large-area land-cover mapping in semi-arid ecosystems: Implications for global monitoring." Remote Sensing of Environment 115 (2011), 2445-2459.

Klein, Roberto. M. "Ecologia da flora e vegetação do Vale do Itajaí” Itajaí: Sellowia 32 (1980).

Johansen, Kasper, and Phinn, Stuart. "Mapping structural parameters and species composition of riparian vegetation using IKONOS and Landsat ETM+ data in Australian tropical savannahs." Photogrammetric Engineering and Remote Sensing 72(1) (2006), 71-80.

Johansen, K., Coops, Nicholas C., Gergel, Sarah E., and Stangec, Yulia. "Application of high spatial resolution satellite imagery for riparian and forest ecosystem classification." Remote Sensing of Environment 110 (2007), 29-44.

Liebsch, Dieter, Marques, Marcia C.M., and Goldenberg, Renato. "How long does the Atlantic Rain Forest take to recover after a disturbance? Changes in species composition and ecological features during secondary succession.” Biological Conservation, Kidlington 141(6) (2008), 1717-1725.

Liesenberg, Veraldo, Boehm, Hans-Dieter V., and Gloaguen, Richard. "Spectral variability and discrimination assessment in a tropical peat swamp landscape using CHRIS/PROBA data". GIScience \& Remote Sensing 47 (2010), 541-564.

Lu, Dengsheng, Li Guiying, Moran Emilio, and Kuang, Wenhui. "A comparative analysis of approaches for successional vegetation classification in the Brazilian Amazon." GIScience \& Remote Sensing 51(6) (2014), 695-709.

Mantovani, Waldir. "Dinâmica da floresta pluvial Atlântica." In: Anais do IV Simpósio de Ecossistemas Brasileiros." Águas de Lindóia. São Paulo, ACIESP, 1998.

Matthews, Emily, Payne, Richard, Rohweder, Mark, and Murray, Siobhan. Pilot analysis of global ecosystems: forest ecossystems. Washinton D.C.: World Resources Institute, 86p, 2000.

Meneses, Paulo R., and Netto, José S. Sensoriamento Remoto: Reflectância dos alvos naturais. Brasília, DF: UnB; Planaltina: Embrapa Cerrados, 2002.

Oliver, Chadwick. D., and Larson, Bruce. C. Forest Stand Dynamics. New York: McGraw-Hill, 1990.

Orihuela-Belmonte, Edith, De Jong, Bernardus. H. J., Mendonza-Vega, J., Van der wal, H., PazPellat, Fernando, Soto-Pinto Lorena, and Flamenco-Sandoval, Alejandro. "Carbon Stocks and 
Accumulation Rates in Tropical Secondary Forests at the Scale of Community, Landscape and Forest Type." Agriculture, Ecosystems and Environment 171 (2013), 72-84.

Prates-Clark, Cássia C., Lucas, Richard M., and Santos, João R., "Implications of Land-Use History for Forest Regeneration in the Brazilian Amazon." Canadian Journal of Remote Sensing 35 (2009): 534-553.

Piazza, Gustavo A., Vibrans, Alexander C., Liesenberg, V., and Refosco, Júlio C. "ObjectOriented and pixel-based classification approaches to classify tropical successional stages using airborne high-spatial resolution images". GIScience \& Remote Sensing 53(2) (2016), 206-226.

Piazza, Gustavo. A. "Processamento digital de imagens de alta resolução espacial com enfoque na classificação dos estágios sucessionais iniciais da Floresta Ombrófila Densa em Santa Catarina.” Dissertation (Master), Fundação Universidade Regional de Blumenau, 2014.

Ponzoni, Flávio. J., Shimabukuro, Yosio. E., Kuplich, Tatiana. M. "Sensoriamento remoto da vegetação" 2nd edition. Oficina de Textos, 2012.

Reis, Ademar, Reis, Maurício S., and Fantini, Alfredo. C. Manejo do palmiteiro (Euterpe edulis) em regime de rendimento sustentado. Universidade Federal de Santa Catarina (UFSC), 1995.

Ribeiro, Milton C., Metzger, Jean P., Martensen, Alexandre C., Ponzoni, Flávio J., and Hirota, Márcia M. "The Brazilian Atlantic Forest: How much is left, and how is the remaining forest distributed? Implications for conservation.” Biological Conservation 142 (2009), 1141-1153.

Schuch, Cristiano., Siminski, Alexandre, and Fantini, Alfredo C. "Usos e potencial madeireiro do jacatirãoaçú (Miconia cinnamomifolia (De Candolle) no litoral de Santa Catarina." Floresta (UFPR. Impresso) 38 (2008), 735-741.

Sette, Priscila G. C. "Análise da textura de imagem de alta resolução para classificação de estágios sucessionais de Mata Atlântica do sul da Bahia." Dissertation (Master), Instituto de Geociências - Universidade Federal de Minas Gerais, 2009.

Silva, Ricardo. D., Galvão, Lênio S., Santos, João. R., Silva Camila V., and Moura, Yhasmin M. "Spectral/textural attributes from ALI/EO-1 for mapping primary and secondary tropical forests and studying the relationships with biophysical parameters." GIScience \& Remote Sensing 51 (2014), 677-694.

Siminski, Alexandre. "A floresta do futuro: conhecimento, valorização e perspectivas de uso das formações florestais secundárias no estado de Santa Catarina.” Thesis (Dostorate). Florianópolis: Univerisdade Federal de Santa Catarina (UFSC). 2009.

Siminski, Alexandre, Mantovani, Marcelo, Reis Mauricio. S., and Fantini, Alfredo C. "Sucessão Florestal Secundária no município de São Pedro de Alcântara, Litoral de Santa Catarina: Estrutura e diversidade." Ciência Florestal 14 (2004), 21-33.

Siminski, Alexandre, Fantini, Alfredo, C., and Reis, Mauricio. S. "Classificação da vegetação secundária em estágios de regeneração da Mata Atlântica em Santa Catarina." Ciência Florestal, Santa Maria, 23(3) (2013), 369-378.

Souza, Agostinho. L., Hosokawa, Roberto T., Kirchner, Flávio F., and Machado, Sebastião A. "Análises multivariadas para manejo de floresta natural na Reserva Florestal de Linhares, Espírito Santo: análises de agrupamento e discriminante.” Revista Árvore 14(2) (1990), 85-101.

Stagakisa, Stavros, Markos, Nikos, Sykioti, Olga, and Kyparissis, Aris. "Monitoring canopy biophysical and biochemical parameters in ecosystem scale using satellite hyperspectral imagery: an application on a Phlomis fruticosa Mediterranean ecosystem using multiangular CHRIS/PROBA observations." Remote Sensing of Environment 114 (2010), 977-994. 
Steininger, M. K. "Satellite estimation of tropical secondary forest above ground biomass: data from Brazil and Bolivia.” International Journal of Remote Sensing 21 (2000), 1139-1157.

Teixeira, Ana M. G., Soares-Filho, Britaldo S., Freitas, Simone R., and Metzger, Jean P. "Modeling landscape dynamics in an Atlantic Rainforest region: Implications for conservation." Forest Ecology and Management 257 (2009), 1219-1230.

Tenenbaum, David E., Yang, Yun, and Zhou, Weiqi. A "Comparison of Object-Oriented Image Classification and Transect Sampling Methods for Obtaining Land Cover Information from Digital Orthophotography." GIScience \& Remote Sensing 48 (2013), 112-129.

Thenkabail, Prasad S, Smith, Ronald B., and Pauw, Eddy. "Hyperspectral vegetation indices and their relationships with agricultural crop characteristics." Remote Sensing of Environment 71 (2000), 158-182.

Tucker, Compton J.; and Townshend, John R. G. "Strategies for monitoring tropical deforestation using satellite data." International Journal of Remote Sensing 21(6) (2000), 14611471.

Vibrans, Alexander. C. “A cobertura florestal da Bacia do Rio Itajaí: elementos para uma análise histórica." Thesis (Doctorate). Florianópolis: Universidade Federal de Santa Catarina, 2003.

Vibrans, Alexander C., McRoberts, Ronald E., Moser, Paolo, and Nicoletti, Adilson L. "Using satellite image-based maps and ground inventory data to estimate the remaining Brazilian Atlantic forest in Santa Catarina." Remote Sensing of Environment 130 (2013), 87-95.

Vibrans, Alexander. C., Sevegnani, Lucia., Gasper, André L., and Linger, Débora. V. Diversidade e Conservação dos remanescentes florestais: Inventário Florístico Florestal de Santa Catarina 1. 1st edition. Blumenau: Edifurb, 2012.

Vibrans, Alexander C., Sevegnani, Lucia, Lingner, Débora V., Gasper, André L., and Sabbagh, Shams. "Inventário florístico florestal de Santa Catarina (IFFSC): aspectos metodológicos e operacionais." Colombo 30 (64) (2010), 291-302.

Vieira, Ima C. G, Almeida, Arlete S., Davidson, Eric A., Stone, Thomas A., Carvalho, Cláudio J. R., and Guerrero, J. B. "Classifying sucessional forrests using Landsat spectral properties and ecological cherecteristics in eastern Amazônia.” Remote Sensing of Environment 87 (2003), 470481.

Wang, Xiao-Qing, Li, Zeng-Yuan, Liu, Xing-E, Deng, Guang, and Jiang, Ze-Hui. Estimating stem volume using QUICKBIRD imagery and allometric relationships for open Populus xiaohei plantations. Journal of Integrative Plant Biology 49(9) (2007), 1304-1312.

Wulder, Michael A., Hall, Ronald J., Coops, Nicholas C., and Franklin Steven E. "High spatial resolution remotely sensed data for ecosystem characterization." Bioscience 54 (2004), 511-521.

Zhang, Caiyun, and Xie, Zhixiao. "Combining Object-Based Texture Measures with a Neural Network for Vegetation Mapping in the Everglades from Hyperspectral Imagery." Remote Sensing of Environment 124 (2012), 310-320.

Zhang, Caiyun, Xie, Caiyun, and Selch, Donna. "Fusing LIDAR and digital aerial photography for object-based forest mapping in the Florida Everglades" GIScience \& Remote Sensing 50 (2013), 562-573.

Recebido em fevereiro de 2015.

Aceito em abril de 2016. 